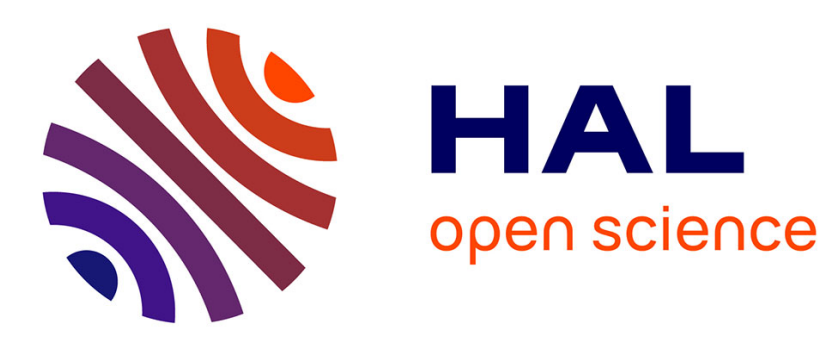

\title{
La protection contre l'incendie des reboisements domamaux du Nord-Ouest de l'Hérault
}

\author{
Jean Prax
}

\section{To cite this version:}

Jean Prax. La protection contre l'incendie des reboisements domamaux du Nord-Ouest de l'Hérault. Revue forestière française, 1951, 4, pp.281-291. 10.4267/2042/27774 . hal-03380166

\section{HAL Id: hal-03380166 https://hal.science/hal-03380166}

Submitted on 15 Oct 2021

HAL is a multi-disciplinary open access archive for the deposit and dissemination of scientific research documents, whether they are published or not. The documents may come from teaching and research institutions in France or abroad, or from public or private research centers.
L'archive ouverte pluridisciplinaire HAL, est destinée au dépôt et à la diffusion de documents scientifiques de niveau recherche, publiés ou non, émanant des établissements d'enseignement et de recherche français ou étrangers, des laboratoires publics ou privés. 
PROTECTION CONTRE L'INCENDIE DANS L'HÉRAULT

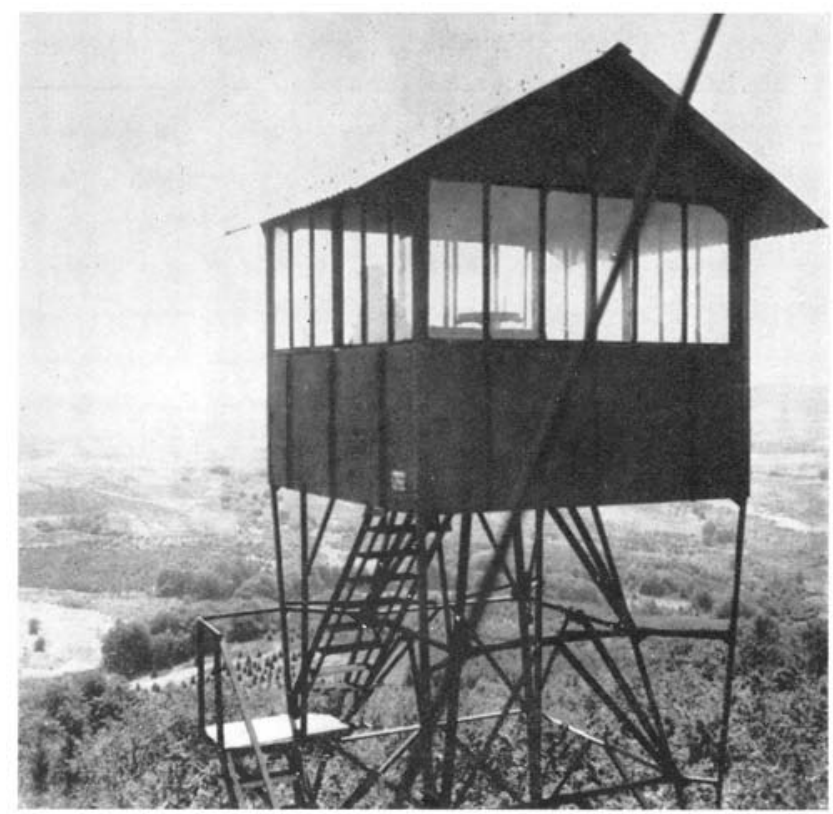

Observatoire de guet installé à la cote 1048 clans la Série Dale de la Salvetat-sur-Agout. - Vue de l'habitacle vitré.

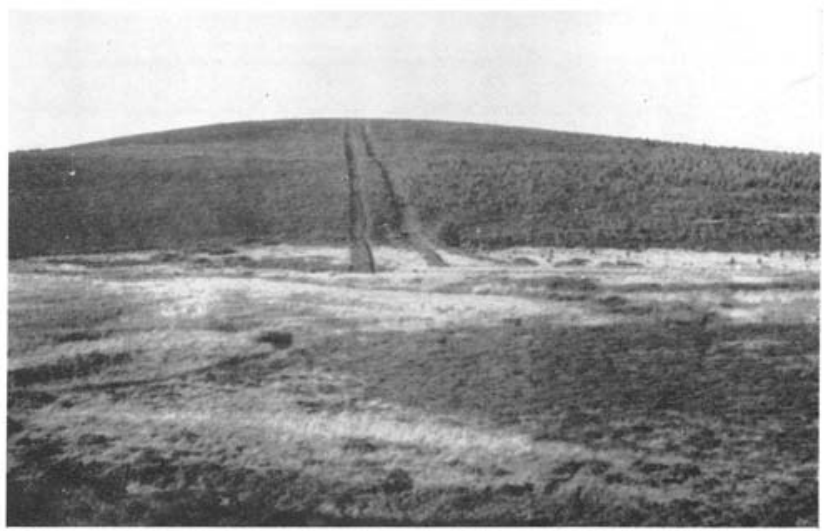

Allée garde-feu en bordure d'une plantation de résineux. Deux bandes latérales ont été labourées.

La bande médiane sera incinérée par temps favorable. 


\title{
LA PROTECTION CONTRE L'INCENDIE DES REBOISEMENTS DOMANIAUX DU NORD-OUEST DE L'HERAULT
}

\author{
Indice bibliographique: F 43.1 (44.84)
}

Les grands incendies de forêts, qui ont ravagé au cours de ces dernières années la région des Landes de Gascogne et le Sud-Est de notre pays, ont attiré l'attention sur un problème difficile, mais dont la solution est rendue d'autant plus urgente que la surface des peuplements résineux artificiels sensibles au feu est actuellement en cours d'extension rapide grâce au Fonds Forestier National.

Je veux parler de la Protection des massifs forestiers contre l'incendie et de la lutte contre les feux de forêt.

A vrai dire, il s'agit là d'une question qui, en France, à l'inverse de la plupart des grands pays forestiers du monde, ne ressort pas complètement des attributions de notre Administration.

Il est permis de le regretter (I) car, si l'on en juge par les résultats, il est bien dificile de s'estimer satisfait par l'organisation actuelle telle qu'elle est issue de la loi du 26 mars I924 et des textes subséquents.

L'expérience montre en effet que dans les régions où la forêt ne joue qu'un rôle économique réduit, par suite de son état de dégradation ou en raison de la faible valeur économique de ses produits, la protection des peuplements contre l'incendie n'intéresse pas les populations et par suite ne suscite de la part des pouvoirs publics locaux, chargés par la loi des mesures de défense, qu'un intérêt de circonstance.

Tel est le cas de la plupart des départements méditerranéens et tel sera sans doute le cas de bon nombre de régions agricoles pauvres où la mise en valeur des terres incultes par le boisement est appelée à se développer.

Ici et là la forêt, soit dégradée, soit en cours de constitution, ne fournit pas un revenu immédiat capable de vaincre l'indifférence des populations.

Le Forestier est seul à comprendre la nécessité de la protéger

(r) Voir à ce sujet l'opinion de P. Galloy, Président de la Fédération Girondine des Associations de D.F.C.I., in Cahier des Ingénieurs Agronomes, $4^{\bullet}$ trimestre 1949. 
pour lui permettre soit de se reconstituer en vue d'assurer la conservation du sol, soit de fournir des produits rentables à une échéance qui peut paraître lointaine au commun des mortels.

C'est en présence d'une telle situation et pour répondre à des préoccupations de cet ordre que le Service Forestier dẹ l'Hérault a organisé dès I947 la défense contre les incendies.des massifs domaniaux du Nord-Ouest du département, " agissant en la circonstance, comme un propriétaire particulier pour la défense de son patrimoine $)$.

Financé par le budget d'Equipement, le projet de Défense des Forêts contre l'Incendie (D.F.C.I.) a été réalisé en trois tranches successives de 1947 à I949. Une tranchè complémentaire est en cours d'exécution sur l'exercice 1950 .

Les réalisations ainsi obtenues, bien qu'elles ne comportent aucune innovation, sont susceptibles d'intéresser les forestiers chargés de l'élaboration des plans départementaux de Défense contre les Incendies de Forêt.

C'est à ce titre que nous croyons devoir fournir quelques renseignements aux lecteurs de la Reviue Forestière.

\section{LE REBOISEMENT ET LE PROBLÈME DES INCENDIES DANS LES CÉVENNES MÉRIDIONALES}

Il parait d'abord nécessaire de situer le problème de la D.F.C.I. dans le cadre forestier local.

Les premiers travaux de boisements domaniaux ont été entrepris dans l'Hérault, à la suite des lois de I86o et de I882 sur la Restauration des terrains en montagne. L'Etat a acquis à ce titre 26.000 ha environ de terrains dans les périmètres de l'Orb, du Jaur, de l'Agout, de l'Hérault et de la Lergue.

Les deux tiers de ces Séries Domaniales de reboisement sont situés dans la zone Nord et Nord-Ouest du département, sur les chaînes du Somail, de l'Espinouse, des Avants-Monts et des Monts d'Orb, désignées sous le terme d'ensemble de Cévennes méridionales.

Il s'agit de terrains siliceux anciens (schistes et gneiss) entre $500 \mathrm{~m}$. et $1.100 \mathrm{~m}$. d'altitude, avec une pluviosité variant de 1.000 à $\mathrm{I} .500 \mathrm{~mm}$, dans une zone à influences méditerranéennes marquées. Les précipitations assez mal réparties, sont faibles pendant la période d'été (juillet à septembre) et les sécheresses hivernales ne sont pas rares.

De plus, outre ces facteurs climatiques défavorables, le danger d'incendie qui menace les boisements artificiels des périmètres de restauration se trouve aggravé par:

- l'abondance de la végétation herbacée ou frutescente formée surtout par des espèces pyrophytes (callune, bruyère cendrée, ge- 
nêt à balais, fougère aigle) qui résulte de la nature siliceuse du sol.

- la pratique de l'incinération propre aux populations pastorales et utilisée depuis un temps immémorial en vue de renouveler l'herbe des pâturages à mouton.

- le fait que nos plantations, destinées à produire des bois tendres, comportent une forte proportion de résineux (Douglas, Epicéa, Sapin, Cèdre, Pins divers) très sensibles à l'incendie tant que le massif n'est pas fermé.

Il est vrai qu'une fois la couverture vivante de pyrophytes éliminée par le couvert (soit entre 30 et 40 ans), les peuplements de résineux, sauf peut-être les pins, se montrent parfaitement résistants à l'incendie.

Le problème revient donc à assurer leur protection jusqu'à ce qu'ils aient atteint cet âge limite.

L'expérience prouve par ailleurs que les incendies, contre lesquels nous devons lutter et qui sont provoqués le plus souvent par l'incinération des parcours à moutons, sont rapidement maitrisés sans gros dégâts, sous réserve:

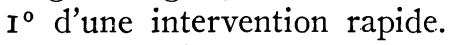

$2^{\circ}$ d'un cloisonnement aussi serré que possible des massifs au moyen d'allées garde-feu ou de chemins, maintenus en parfait état de propreté.

$3^{\circ}$ de l'incinération préventive en saison favorable des landes ou terrains de pâturage en bordure des garde-feu de périmètre.

L'intervention rapide en cas de sinistre implique, d'une part, un système de détection organisé des fumées suspectes et d'autre part la mise à disposition rapide des sauveteurs de moyens de transport et de lutte motorisés.

Enfin l'entretien des garde-feu et des chemins de terre, à des prix de revient acceptables, dans une région où la main-d'œuvre est rare et chère, exige l'emploi d'engins mécaniques à grand rendement.

Telles sont les idées maitresses qui ont inspiré l'organisation de D.F.C.I. mise sur pied au cours de ces dernières années.

Suivant une terminologie généralement admise, je distinguerai:

- les moyens de lutte active,

- les moyens de défense préventifs.

\section{A. - Défense active}

I. - Dispositif de détection et d'alerte. - Il comprend:

a) Observatoires ou postes de guet.

Les boisements domaniaux du N.-O. de l'Hérault à couvrir par le réseau de détection occupent un quadrilatère de $40 \mathrm{~km}$ de long 
sur $35 \mathrm{~km}$ de largeur, soit une surface d'environ $\mathrm{I} 50.000$ ha qui équivaut au quart du département. Ils s'étendent, avec des dénivellations de 500 à I. Ioo m., sur deux chaînes orientées grosso modo E.-W. séparées par un sillon profond, où coule le Jaur. Le relief, sans être extrêmement tourmenté, n'en offre pas moins de sérieuses difficultés pour le repérage des fumées. On est loin des conditions optima de la plaine landaise où aucun obstacle ne gêne le champ visuel. Par contre, la présence de pitons rocheux en situation naturellement dominante permet de réduire la hauteur et, par suite, le prix de revient des observatoires. C'est ainsi que nos plateformes d'observation ont été construites à $6 \mathrm{~m}$. de haureur au-dessus du sol naturel contre 25 à $30 \mathrm{~m}$. dans les Landes.

Les prévisions du projet primitif (I2 postes de guet) ont dû être réduites en raison de la hausse des prix, intervenue entre temps, de sorte que 6 stations de détection seulement ont été construites. L'expérience montre d'ailleurs que, compte tenu des conditions locales de relief, cette densité (I poste pour $250 \mathrm{~km}^{2}$ en moyenne) est suffisante.

Chaque observatoire est essentiellement constitué:

- par un support en forme de tronc de pyramide construit en fer cornière, ancré de la base dans des dés de béton. Hauteur $6 \mathrm{~m}$. Grande base $3 \mathrm{~m}$. $50 \times 3 \mathrm{~m}$. 50 .

- par une cabine métallique couverte et vitrée, dont le plancher repose sur le support précédent. Dimensions: $2 \mathrm{~m} .50 \times 2 \mathrm{~m} .50$ et $2 \mathrm{~m}$. de hauteur.

- par un escalier métallique à 3 volées tournant autour du support et donnant accès à la cabine par une trappe ouverte dans le plancher.

A l'usage, il s'avère en effet indispensable de construire un habitacle parfaitement à l'abri des intempéries.

Pendant la période critique, en matière d'incendie, qui va de janvier à avril, le personnel, chargé de la surveillance, se trouve souvent exposé à des températures très froides dont l'effet est aggravé par la violence des vents soufflant de façon à peu près continue, sur les crêtes. Il n'est pas jusqu'à la période d'été qui ne présente souvent elle aussi des conditions assez dures.

Par suite, à la différence des observatoires landais que j'ai eı l'occasion de visiter, l'observateur est ici protégé à l'intérieur de la cabine par des panneaux vitrés coulissants à armature métallique.

Au centre de la plateforme se trouve une table métallique circulaire supportant un disque gradué, orienté suivant le Nord Lambert et une alidade. De plus, un plan de la zone à surveiller, sur lequel un rapporteur a été fixé, permet au guetteur de reporter les gisements résultant de ses visées et de situer ensuite sur le terrain, au moyen de jumelles à prismes, les fumées qu'il a repérées.

Un appareil téléphonique relié au bureau de poste voisin assure 
les liaisons avec le poste central de D.F.C.I. et le personnel forestier.

L'ensemble des appareils de repérage ou de liaison est enfermé dans des carters métalliques cadenassés.

b) Poste central de D.F.C.I.

Installé au cœur du massif domanial, dans la Maison Forestière de Combesalat sur la Commune de la Salvetat-sur-Agout, il centralise tous les renseignements fournis par les observatoires.

A cet effet, une permanence est assurée par le personnel forestier de la Brigade, pendant les périodes critiques d'hiver et d'été:

Le responsable a une quadruple mission:

I $^{\circ}$ Centraliser, coordonner et au besoin provoquer les renseignements des observatoires.

$2^{\circ}$ Situer exactement les fumées suspectes et apprécier si l'incendie (il s'agit le plus souvent de feux pastoraux ou culturaux sur landes ou genètières) menace directement un massif boisé.

$3^{\circ}$ Fournir, en cas d'incendie déclaré. tous renseignements sur la marche du feu.

Alerter les Centres de Secours permanents.

Pour remplir cette mission, le responsable dispose:

- des moyens de liaison téléphonique (central à plusieurs directions) et automobile (Jeep de liaison).

- d'un plan au I/50.000 (calque de la carte d'E. M.) de la zone couverte par le réseau de protection, sur lequel tous les massifs forestiers ont été reportés. Chaque carré formé par le carroyage Lambert est repéré au moyen de coordonnées rectangulaires.

A l'emplacement de chaque observatoire, un rapporteur circulaire a été fixé qui permet, par un jeu de ficelles, de reporter immédiatement sur le plan les gisements indiqués par les guetteurs.

Le recoupement des directions ainsi matérialisées situe l'incendie avec une précision très suffisante.

\section{II. - Organisation de la lutte active.}

Basée sur trois centres de secours permanents installés en Maison Forestière:

- I à la Maison Forestière de Combesalat sur la commune de la Salvetat-sur-Agout.

- I A lá Maison Forestière Alexandre sur la commune des Verreries de Moussans.

- I à la Maison Forestière d'Agoudet sur la commune de Cambon.

L'équipement de chacun de ces centres comporte:

- Un camion Dodge des surplus type command car, à 2 essieux moteurs, portant une citerne de 800 litres en tôle d'acier et 
un groupe moto-pompe $3 \mathrm{CV}$, susceptible de fournir un débit réglable entre $\mathrm{r} .000$ et 6.000 litres à l'heure, mais fonctionnant normalement à 2.000 litres-heure pour une pression de $6 \mathrm{~kg} / \mathrm{cm}^{2}$. L'ensemble de cet équipement, qui comprend $30 \mathrm{~m}$. de tuyaux caoutchouc montés sur dévidoir et $40 \mathrm{~m}$. de tuyaux de refoulement en toile, a été construit par la Maison Guinard.

La dotation de chaque camion comporte de plus 6 seaux-pompes dórsaux "Indian ) en caisse bois pour faciliter le transport, et des outils divers (haches, serpes, pioches).

- Un dépôt de matériel: seaux-pompes ( Indian ), haches, serpes, pioches, etc...

Le personnel de chaque centre est constitué par un chauffeur permanent, occupé hors de la période d'incendie à la conduite des tracteurs, les préposés et les ouvriers des chantiers forestiers travaillant à proximité.

De plus, dans la plupart des localités voísines, les maires ont dressé une liste de sauveteurs 'plus ou moins bénévoles chargés de renforcer le personnel assez réduit des Centres de secours, en cas d'incendie déclaré de quelque importance.

\section{B. - Moyens De DÉfense PRÉventifs}

Je n'insisterai pas sur la nécessité impérieuse d'une éducation du public, problème délicat d'ordre surtout psychologique, à propos duquel des réflexions pertinentes et autorisées ont paru ici même, il y a peu de temps (Dugelay. Réflexions morales sur les Incendies de Forêts, in Revue Forestière de septembre 1950).

J'indiquerai toutefois que, dans le département de l'Hérault, une action dans ce sens a été menée depuis plusieurs années auprès des enfants des écoles sous l'égide de la Commission Départementale de Reboisement et sous l'énergique impulsion de $M$. le Conservateur Prioton qui a publié à cet effet une brochure illustrée de propagande "Les Incendies de forêts et Garrigues Méditerranéennes ) où sont condensées toutes les notions essentielles sur la question.

Toutefois, cette mobilisation de la ( conscience ) publique ne peut manifester ses effets que dans un avenir relativement lointain et elle ne saurait dispenser le Service d'agir dans l'immédiat sur le terrain.

C'est précisément sous l'aspect d'une véritable " Organisation du terrain » au sens militaire du terme, qu'il convient de considérer l'ouverture et l'entretien en état du réseau de chemins forestiers et d'allées garde-feu. En cas d'incendie, les sauveteurs doivent, en effet, pouvoir trouver sur le terrain des " points d'appui » préparés à l'avance, le long desquels une ligne d'arrêt ou de contrôle sera établie. 
C'est dans ce but qu'ont été ouverts:

- des pare-feux périmétraux: Ils séparent les boisements domaniaux des landes privées ou communales, source la plus fréquente des incendies. Une fois mises en état, ces allées de périmètre servent de base de départ pour l'incinération préventive, en saison et par temps favorable, des parcours limitrophes. Ces écobuages " dirigés " sont réalisés, avec l'accord des riverains, au moyen de l'incinérateur " Flame Gun » fonctionnant au pétrole ou au Kérosène. Cette opération assez délicate, à ne réaliser qu'en dehors des périodes de sécheresse et par temps calme, exige un personnel nombreux, muni du matériel d'extinction habituel (seauxpompes, citerne, moto-pompe, etc.), de manière à contrôler constamment la propagation du feu.

- des pare-feux de cloisonnement: Ils compartimentent le massif en parcelles de surface variable (Io à 39 ha). Leur but est de servir de base à un contre-feu destiné à stopper un incendie qui aurait pénétré à l'intéricur du boisement.

Pour $75 \%$ de leur surface, l'ouverture et l'entretien de ces allées garde-feu sont réalisés dans le périmètre de l'Agout au moyen d'engins mécaniques tractés.

Le matériel utilisé à cet effet est le suivant:

- Un tracteur Clétrac à chenille Diesel zo C.V.

- Un tracteur Ford-Ferguson I8-22 C.V.

Quant aux engins de travail proprement dits, ils sont de divers types:

- rouleaux débroussailleurs landais.

- charrues à soc.

- charrues à disques de $65 \mathrm{~cm}$ Mac Cormick.

- pulvériseurs à disques.

Ce matériel est d'ailleurs avantageusement employé à des travaux de repeuplement en régie (labours ou préparation du sol pour semis ou plantations, etc...).

De plus, un appareil de débroussaillement fabriqué par Ferguson " Le Rotavator " qui, au cours de différentes démonstrations, a donné des résultats remarquables, est en cours d'acquisition.

\section{COUT. ET FRAIS DE FONCTIONNEMENT}

Il peut être intéressant, à maints égards, de préciser le prix de revient de chacun des éléments de l'organisation de D.F.C.I. ainsi mise sur pied. Toutefois, remarque est faite, que l'acquisition du matériel s'est échelonnée sur 3 exercices (I947-1948-1949) et a coincidé avec une période d'instabilité monétaire particulièrement marquée. 
Les dépenses d'investissement réalisées au 3I décembre I949 se répartissaient comme suit:

I. - Construction et mise en place de 6 observatoires métalliques de guet, y compris tous les accessoires de repérage, etc... ...........

2. - Frais d'installation des lignes téléphoniques desservant les 6 observatoires (frais payés à l'Administration des P.T.T.). ..........

3. - Achat et Equipement avec citerne moto-pompe, en vue de la lutte contre l'incendie, de 3 camions Dodge command car avec remorque et

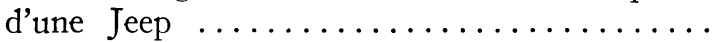

4. - Achat de 2 tracteurs (un chenillard Clétrac et un Ford Ferguson à roues) et des appareils de travail divers (charrues, débroussailleurs, etc.).

5. - Achat de matériel léger: seaux-pompes, battafeux, etc... (La dépense ci-contre ne comporte pas le matériel fourni directement par l'Administration tel que certains seaux pompes " Indian ) et les incinérateurs. De plus, la majeure partie du matériel léger est en cours d'acquisition sur la tranche 1950$) \ldots \ldots \ldots \ldots \ldots$

6. - Frais d'études et achats divers ........ 219.798

TOTAL

La surface soumise au régime forestier (Forêts domaniales ou communales et Séries domaniales de reboisement) protégée par l'Orvanisation de D.F.C.I. est en gros de 20.000 ha.

Les dépenses d'investissement se montent donc à $500 \mathrm{fr}$. environ par ha. Ce chiffre, relativement faible, pourrait encore être abaissé dans une région à plus forte densité forestière puisque les terrains soumis n'occupent ici que $\mathrm{I}_{5} \%$ de la surface totale couverte par le réseau de détection.

A vrai dire, comme je l'indiquerai plus loin, si le dispositif de surveillance est, dans son état actuel, suffisant, les moyens de lutte active (centres de secours et matériel motorisé) ainsi que les engins mécanisés pour la création d'allées garde-feu exigeraient, pour ètre pleinement efficaces, d'être notablement renforcés.

Quant aux frais de fonctionnement fixes annuels, on peut, schématiquement et compte tenu des prix actuels, en donner la répartition suivante: 
I. - Salaire de 6 ouvriers civils assurant le Service de guet pendant I50 journées : $120.000 \times 6 \ldots$ (du I5 janvier au $I^{\text {er }}$ avril et du I 5 juillet au 30 septembre).

2. - Salaire annuel de 3 chauffeurs assurant la conduite des camions citernes et des tracteurs

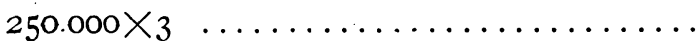

3. - Frais d'abonnement et communications téléphoniques à régler à l'Administration des P.T,T. . 250.000

4. - Frais d'entretien du matériel motorisé ...... 300.000

$$
\text { TOTAL }
$$

2.020 .000

$\mathrm{Ne}$ sont pas compris dans l'évaluation ci-dessus les frais résultant de la lutte proprement dite contre les incendies déclarés, lorsque cette lutte exige la mise en œuvre de moyens en personnei et matériel extérieurs à l'organisation de D.F.C.I. Tels quels, ils font ressortir le prix de revient du fonctionnement de cette organisation à Ioo fr. environ par hectare.

\section{LES RÉSULTATS}

A peine organisé, le dispositif de D.F.C.I., que nous venons de décrire, a été mis à l'épreuve dans des conditions particulièrement dures.

Dans toute la région méditerranéenne, l'été I950 s'est révélé anormalement sec, comme en témoignent les grands incendies de Provence.

Après un hiver plutôt humide, la sécheresse dans notre région a brutálement débuté en mai et jusqu'à fin septembre les précipitations ont été pratiquement inexistantes (I).

Le réseau de détection a été mis en fonctionnement le $\mathrm{I}^{\text {er }}$ juillet et, sauf quelques rares interruptions par journées de brouillard ou de pluie, n'a été arrêté qu'aux premiers jours d'octobre.

(I) Les précipitations observées à la M. F. de Combesalat (I.000 m. d'altitude) $\mathrm{du} \mathrm{I}^{\mathbf{e r}}$ janvier au 30 septembre 1950 ont été les suivantes (en $\mathrm{mm}$.) :

Les chiffres de la seconde ligne indiquent la moyenne des précipitations observées à la Salvetat-sur-Agout pendant la décade 1935-45.

\begin{tabular}{|c|c|c|c|c|c|c|c|c|c|}
\hline Janv. & Févr. & $\underset{\text { Mars }}{-}$ & Avril & Mai & $\underline{\text { Juin }}$ & Juill. & Août & Sept. & Total \\
\hline \multicolumn{10}{|c|}{ M. F. Combesalat (1950) } \\
\hline 70,1 & 165,2 & I 39,7 & 125 & 35,4 & 23,5 & 23,5 & $2 \mathrm{I}, 7$ & 46,6 & 650,70 \\
\hline 36,3 & & 128 & 12 & 142,3 & 69,7 & 53,2 & 82,05 & 140,5 & 023,15 \\
\hline
\end{tabular}


Durant cette période:

- I26 appels d'alerte ont été reçus par le Poste central de D.F.C.I. à la Maison Forestière de Combesalat.

- Sur ce nombre, 6 incendies seulement ont motivé la mise en œuvre des moyens de défense active. Il est vrai que, pour 2 d'entre eux la lutte a duré plusieurs jours par suite des difficultés rencontrées dans la surveillance des foyers éteints et des reprises continuelles dues à une couverture morte extrêmement inflammable.

- Pour tous ces incendies, sauf un, allumé vraisemblablement de nuit, l'alerte a été donnée dans un laps de temps variant entre I5 minutes et un heure après la mise à feu probable.

- Malgré des conditions climatiques très défavorables, les peuplements soumis n'ont eu à supporter que des dégâts négligeables. Aucun peuplement résineux issu de plantations récentes (moins de 30 ans) n'a été atteint. Dans tous les cas, le feu, qui avait pris naissance dans la lande, a été soit arrêté loin des lisières, soit maintenu, puis stoppé en bordure des peuplements.

\section{Conclusions}

L'expérience acquise au cours de ces dernières années permet, d'ores et déjà, de tirer certaines leçons :

C'est tout d'abord la possibilité d'un très large emploi pour la D.F.C.I. des véhicules et des engins motorisés même en région de moyenne montagne.

Les véhicules tout terrain des différents types utilisés par l'Armée Américaine offrent à cet égard des possibilités insoupçonnées. Il nous est arrivé d'arrêter au moyen des citernes moto-pompes, montées sur Dodge command-car, des incendies en terrain varié sur des pentes de 20 à $25 \%$. De plus, ces engins circulent dans n'importe quel chemin de terre et permettent ainsi. d'amener à la fois les sauveteurs et l'eau nécessaire à l'alimentation des seaux-pompes. Pratiquement dans toute la zone granitique du Bassin de l'Agout, entre $650 \mathrm{~m}$. et I.IOO $\mathrm{m}$. d'altitude, il n'est pas de point qui ne leur soit accessible.

Par ailleurs, ce serait une erreur. de croire que l'ouverture et l'entretien de garde-feu par des moyens mécaniques n'est réalisable qu'en plaine: dans toute la région ci-dessus, les trois quarts de nos allées sont entretenues au tracteur. Tel doit être le cas dans la majeure partie du Massif Central.

- Pour obtenir le maximum d'efficacité, les citernes tout terrain doivent être utilisées par groupe d'au moins 2 unités de manière à assurer la continuité de l'attaque et éviter les temps morts nécessaires au réapprovisionnement des réservoirs.

- La liaison par radio entre l'agent chargé sur le terrain de diriger la lutte contre un incendie déclaré et le poste central de 
D.F.C.I. s'avère indispensable. A cet égard, le Service forestier des U.S.A. utilise des appareils de différents types décrits dans "The Fire Control Equipement Handbook » dont les caractéristiques pourraient être avantageusement retenues.

- Enfin, il est regrettable que nous ne disposions pas d'une méthode permettant d'évaluer chaque jour rationnellement, au moyen d'un indice chiffre, les dangers d'incendie, comme cela se pratique ailleurs (I). Ces indications, à mon sens, auraient un gros intérêt plutôt pour le personnel chargé de la D.F.C.I. que pour le public.

Exempte de tout élément subjectif, la fixation de l'indice par une méthode rationnelle permettrait de proportionner la vigilance et la tension d'esprit exigées de ce personnel avec le danger réel couru par les peuplements forestiers.

Décembre 1950 .

J. Prax.

(I) «Les méthodes d'évaluation des dangers d'Incendie Forestier en Amérique du Nord 》, par Paul Reneuve, in Revue Forestière, $\mathrm{n}^{\circ} 6$, juin 1950.

Cf. Les bulletins publiés par le Bureau de météorologie du Ministère des Terres et Forêts de la Province de Québec, sous la direction de G. Oscar Villeneuve $\mathrm{Ph}$. D., en particulier les numéros $7, \mathrm{I} 3$, i5 et 18 . 